\section{In situ force-balance tensiometry}

\author{
G. S. Lapham, D. R. Dowling, W. W. Schultz
}

\begin{abstract}
Although a fundamental physical parameter, surface tension is difficult to measure. Common tensiometry inaccuracy comes from failure to control air-liquid-solid contact conditions, or account for liquid meniscus geometry and buoyancy corrections. This paper describes an in situ tensiometry technique, based on withdrawal of a thin-walled tube from the liquid interface, that enforces a known air-liquid-solid contact condition. This technique can be pursued at any level of experimental hygiene. Experimental results for filtered tap water, an alcohol-water solution, and a surfactant-water solution show that results repeatable to three significant digits are obtained with modest effort for a variety of geometrical parameters.
\end{abstract}

\section{1}

\section{Introduction}

Surface tension is the macroscopic manifestation of a host of molecular phenomena at the interface between two fluids. Surfactants (or surface active agents) may change a liquid's wetting characteristics, and alter surface tension and mass transfer at liquid interfaces. This paper discusses a simple in situ force-balance surface tension measurement technique for both clean and dirty gas-liquid surfaces under ordinary laboratory conditions. The technique is intended for hydrodynamicists who need accurate surface tension measurements with minimal resources.

The usual interpretation of surface tension as the force per unit length exerted across any line lying in the plane of the liquid surface has lead to the development of a variety of force-balance tensiometers. The distinction between surface tension nd surface free-energy per unit area (Ip and Toguri 1994 ) is unessential here. These devices typically rely on placing a solid object (a vertical hollow circular cylinder in this case) into the liquid of interest, determining the length of the macroscopic solid-liquid contact line (hereafter referred to as

\section{Received: 7 October 1997/Accepted: 23 April 1998}

G. S. Lapham, D. R. Dowling, W. W. Schultz

Department of Mechanical Engineering and Applied Mechanics

University of Michigan, Ann Arbor, MI 48109-2125, USA

Correspondence to: G. S. Lapham

417 Pool Road, Biddeford, ME 04005, USA

This research has been supported by the Ocean Engineering Division of the Office of Naval Research under grants N00014-931-0867 and N00014-94-1-0850. the wetted perimeter), and measuring the added force on the object resulting from its contact with the liquid. The interfacial surface tension is then recovered by dividing the measured force by the wetted perimeter. Implicit in these techniques are the assumptions that: i) surface tension does not depend on liquid-surface curvature, ii) the liquid does not apply any force to the submerged portion of the solid beyond hydrostatic pressure, and iii) the angle that the interface makes with the vertical at the contact line is known (usually assumed to be zero for the receding contact angle in the experimental determination of surface tension).

Tensiometry is frequently associated with extraordinary experimental hygiene. Yet the surface tension of ordinary tap-water/air interfaces subject to air-borne particulate contamination is still of interest in many hydrodynamic studies involving large wave tanks or towing basins where high-purity water cannot be used and even daily water changes are not practical. Here, vertical-pull film balances are superior to horizontal or Langmuir film balances (Harkins and Anderson 1937) and surface properties must be monitored in situ because any type of sampling will disturb any intentional or unintentional surfactants. Unlike some tensiometry methods, the technique described here is robust in ordinary laboratory environments and yields consistent results across a variety of wetted-object geometries even when high-purity liquids and clean-room conditions are unavailable.

Accurate surface tension measurements with force balances have proved difficult because the results depend on the contact condition between the object and the liquid interface, the shape of the meniscus, the object's buoyancy, and other possible molecular attraction or repulsion forces between the object and the liquid. These problems have been ignored through simplifying assumptions, treated by ad hoc corrections, mitigated by constraining the measurement technique, or partially corrected by additional measurements. These remedial actions typically prevent in situ measurements or complicate the overall technique reducing its utility and flexibility. More elaborate surface tension measurement techniques have been pursued, but these are application specific and require more resources and process time than is typically available for basic tensiometry.

The purpose of this paper is to present a new tensiometry technique that has advantages over those currently in use. Experiments show the technique is capable of consistent surface tension measurements of commonly available liquids and solutions under ordinary laboratory conditions. The new method is an absolute technique accurate to three 
significant figures across a variety of geometric parameters. Significantly, it does not require knowledge of the liquid's wetting characteristics, empirical or ad hoc corrections, calibration with a test liquid, nor an expensive, specialized apparatus. It requires only the force measurement common to all force-balance methods and may be used in situ in any size basin. This technique has been developed for general purpose use, but with appropriate refinements, it may be exploited at the clean-room precision level as well.

This paper is organized into three additional sections and a summary. A short background summary highlighting current technical inconsistencies is provided in the next section. The third section presents results for a representative traditional technique, the Wilhelmy plate method, that illustrate some of these problems. In the fourth section, the new technique is presented and its consistency is demonstrated by experimental results for several different air-liquid interfaces.

\section{2}

\section{Background}

There are two main techniques for force-balance tensiometry: the Wilhelmy plate, and du Noüy ring methods (Adamson 1990; Gaines 1966; Davies and Rideal 1963). These are discussed in the next two subsections. The final subsection covers other force-balance techniques. Additional information is available in Rusanov and Prokhorov (1996).

\section{1}

\section{Wilhelmy plate method}

There are several variations of the Wilhelmy plate method. All are based on balancing the static forces of surface tension, gravity, and buoyancy acting on a thin plate suspended vertically in the air-liquid interface. Figure 1 shows a crosssectional free body diagram of the active part of the balance while Fig. 2 shows photographs of broadside and end views of

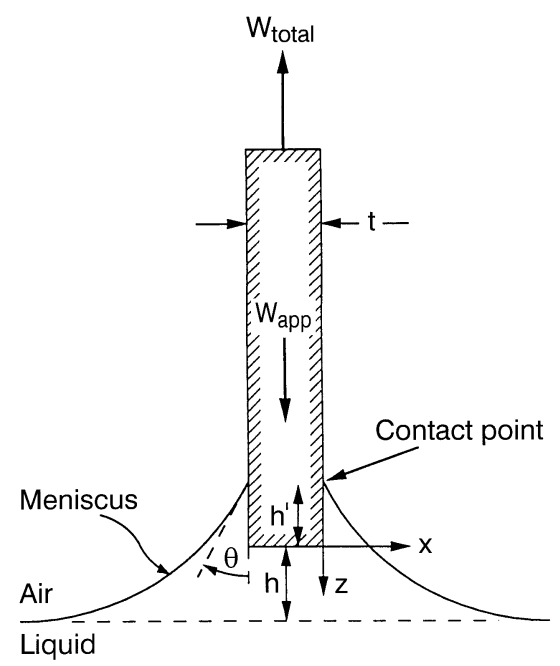

Fig. 1. Free body diagram of the Wilhelmy balance. The total pull on the plate of thickness $t$ and length $L$ (into the page) is balanced by its own weight, the force from surface tension at the contact line, and the negative buoyancy resulting from raising the plate above the mean free surface a distance $h$. The angle from the vertical $\theta$ of the meniscus in contact with the plate is a true contact angle when capillary rise up the plate occurs $\left(h^{\prime} \neq 0\right)$. For the case of no capillary rise, $h^{\prime}$ is zero
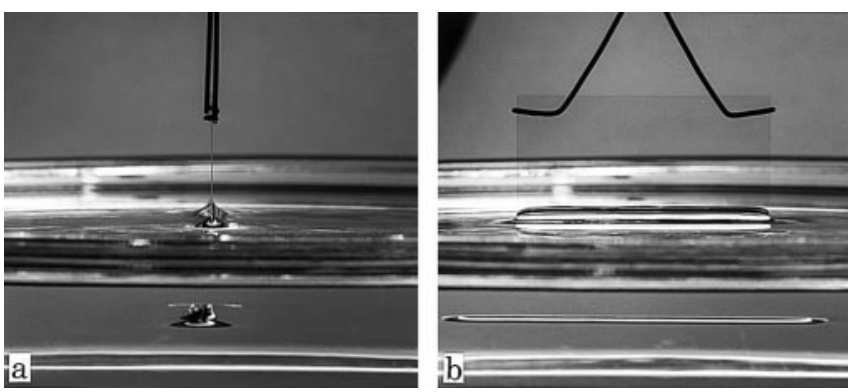

Fig. 2. Photographs of a Wilhelmy plate before detachment: a end view. $\mathbf{b}$ broadside view. The photographs reveal the complexity of the meniscus shape at the plate ends. This complexity and the failure of models to account for it, is a source of error in Wilhelmy plate techniques

a Wilhelmy plate in contact with water. For a known wetted plate perimeter, the experimentalist measures the pull on the balance, and, in some cases, the vertical position of the bottom plate edge relative to the undisturbed free surface. The surface tension $\sigma$ is then determined from (Allan 1958; Jordan and Lane 1964)

$W=W_{\text {total }}-W_{\text {app }}=\int \sigma \cos \theta \mathrm{d} P+\Delta \rho g t L h$.

Here, $W_{\text {total }}$ is the weight registered by a hook balance, $W_{\text {app }}$ is the dry weight of the apparatus (plate and the harness), $P$ is the wetted perimeter of the plate, $\sigma$ is surface tension, $\theta$ is the angle the liquid meniscus makes with the vertical at its point of contact with the plate, $\Delta \rho$ is the density difference between the liquid and the air, $g$ is the gravitational acceleration, $t$ and $L$ are the plate thickness and length (for a rectangular plate $P=2(L+t))$, and $h$ is the height of the bottom of the plate above the undisturbed mean surface. The angle with the vertical $\theta$ is a generalization of the macroscopic or apparent contact angle that remains well-defined at a solid surface discontinuity. The contact angle is the angle defined by Young's equation (Adamson 1990). When a meniscus contacts an object at a corner and no capillary rise up the vertical sides of the object occurs $\left(h^{\prime}=0\right), \theta$ is well defined but the contact angle is not. For the frequently encountered case where some capillary rise occurs $\left(h^{\prime} \neq 0\right), \theta$ is both the contact angle and the angle with the vertical. All the parameters in (1), except $\sigma$, $\theta$, and $h$, are easy to determine accurately. The final term in (1) is called the buoyancy correction. While not large, typically 1 to $10 \%$ of $W_{\text {total }}$, the buoyancy correction is typically not negligible (Gaonkar and Neuman 1984). Note that surface curvature effects indirectly enter (1) through the contact angle $\theta$.

Four variations of the Wilhelmy plate measurement are commonly used: the zero-buoyancy method, the detachment method, the immersion method (Gaines 1966), and the maximum-pull method (La Mer and Robbins 1958; Loglio et al. 1976; Gaonkar and Neuman 1984). In principle, the four variations are similar except for procedural adjustments that simplify the final two terms in (1) so only the first two are considered here. The immersion method gave inconsistent results and the maximum-pull method failed since the meniscus always ruptured before the pull reached a maximum for the thin plates we considered. 
In the zero-buoyancy method, the plate is quasi-statically lowered while keeping the lower plate edge parallel to the plane of the undisturbed liquid surface until the slide first contacts the liquid surface (Padday 1957; Zotova and Trapeznikov 1960; Padday and Russell 1960; Slowinski and Masterton 1961; Pallas and Pethica 1983; Gaonkar and Neuman 1987). The surface tension induced force is then measured under the assumption that $h$ is zero. However, the advancing contact line leads to variability in the contact angle $\theta$. This method is susceptible to significant error if the measured pull at first contact is used. Instead, the plate should be further lowered into the fluid and then withdrawn to the first-touch height to promote better plate wetting (Kawanishi et al. 1970; Lane and Jordan 1970).

In the detachment method (Furlong and Hartland 1979), the plate is quasi-statically pulled from the liquid until the meniscus depicted in Fig. 2 ruptures. Withdrawal of the plate ensures wetting through a receding contact line that drives $\theta$ toward zero. The hope is that $\theta$ approaches zero on the entire plate perimeter as the contact line comes toward the corner near rupture. A thin plate ensures that the buoyancy term is small and that there cannot be much under-cutting of the meniscus before rupture occurs. The detachment method is subject to uncertainty arising from non-repeatability of dynamic meniscus rupture (Padday and Russell 1960; Padday 1969, Loglio et al. 1976).

The most common implementation of either Wilhelmy plate method assumes that the free surface is vertical at the point of attachment (or close enough to justify $\cos \theta=1$ ) and that the buoyancy term can be neglected for a sufficiently thin plate. Hence, Eq. (1) reduces to

$W=W_{\text {total }}-W_{\text {app }}=\sigma P$

The two neglected effects leading to Eq. (2) partially cancel, and this has lead to a lack of consistency between sources about the terms in Eq. (1) that are prudently modified or neglected.

The use of smooth or roughened plates to enhance plate wetting is controversial (Kawanishi et al. 1969). Some investigations generally support roughening plates (Princen 1970; Furlong and Hartland 1979; Gaonkar and Neuman 1987), while others generally oppose it (Jordan and Lane 1964; Lane and Jordan 1970, 1971; Pallas and Pethica 1983). Some investigators motivated by practicality (Pike and Bonnet 1970), like ourselves, merely use the finish obtained on commercially available glassware.

Additional controversy surrounds the necessity of an empirical correction. In some studies (Jordan and Lane 1964; Pike and Bonnet 1970; Lane and Jordan 1971; Furlong and Hartland 1979), as well as the present, a "film deficit" is observed near the plate ends (Fig. 2a). The consequence of this variation in meniscus shape is a perimeter-location dependent value of $\theta$ which causes measured surface tension values to have an unexpected dependence on plate thickness (see Sect. 3 ). This $\theta$ variation is commonly ignored or dismissed (Taylor and Mingins 1975; Orr et al. 1977; Furlong and Hartland 1979; Pallas and Pethica 1983; Sauer and Carney 1990; Palas and Harrison 1990; Mennella and Morrow 1995), or treated with an empirical end or peripheral correction (Padday 1957, 1969; Padday and Russell 1960; Kawanishi et al. 1970; Pike and
Bonnet 1970; Gaonkar and Neuman 1984, 1987). Apparently, this end-correction controversy will not soon be settled either by experiments (Pallas and Pethica 1989, 1991; Gaonkar and Neuman 1991; Pallas and Pethica 1991) or three-dimensional theory (Orr et al. 1975, Orr et al. 1977). The technique described in Sect. 4 does not require any empirical corrections.

\section{2}

\section{du Noüy ring method}

The du Noüy ring method may be the most common forcebalance method. Here, a platinum, wire ring lying in a plane parallel to the liquid surface is submerged in the liquid and then slowly withdrawn while the net fluid force on the ring is measured. In general, the ring with attached liquid meniscus is raised above the mean undisturbed surface until the pull on the ring reaches a maximum. Further raising of the ring causes a reduction of pull. The maximum force, $W_{\max }$ obtained during ring withdrawal can be directly related to the surface tension if the ring is perfectly wetted by the fluid (Harkins and Jordan 1930; Freud and Freud 1930; Cini et al. 1972; Huh and Mason 1975). However, a non-zero contact angle has been found to be important for ring tensiometry (Princen and Mason 1965; Cram and Haynes 1971; Gifford 1978). Hence, one needs a priori knowledge of the contact angle to properly implement the du Noüy ring method, so the Wilhelmy plate is often recommend for work where liquid wetting characteristics are not known (Padday and Russell 1960; Gaines 1966; Gaonkar and Neuman 1984; Adamson 1990).

Flaming the du Noüy ring (or platinum Wilhelmy plates) is a controversial cleaning procedure. Many investigations support flaming (La Mer and Robbins 1958; Lane and Jordan 1970; Cini et al. 1972; Huh and Mason 1977; Furlong et al. 1983), while others (Gaines 1960; Kawanishi et al. 1970; Gaonkar and Neuman 1984) claim that the procedure affects the wetting and thereby affects the surface tension measurement.

Another problem common to the ring (and the plate) is the interpretation of the actual force measurement. Equating the force on a du Noüy ring just before meniscus rupture to any of the points on the force versus height curve produced from theory (such a curve for a different geometry is shown in Fig. 7) to determine the surface tension is not recommended (Padday and Russell 1960; Padday 1969; Huh and Mason 1975). Perhaps the largest single source of misunderstanding in tensiometry is confusing $W_{\max }$ with the experimentally determined point of meniscus detachment. The experimental point of meniscus detachment may occur anywhere along the force versus height curve as determined by film stability and the detailed procedures of the particular force balance method. A typical torsion balance either controls $h$ or $W$, or both, and usually uses the force immediately prior to detachment in the measurement. Some methods are designed to cause meniscus detachment as close as possible to $W_{\max }$, however, the actual proximity is always in doubt once rupture occurs. Certain geometries, like the thin Wilhelmy plates used in this study, cause miniscus rupture before $W_{\max }$ is reached.

The new technique described here avoids these controversies, by allowing the experimenter to document $W$ going through a maximum, by avoiding end effects as in the Wilhelmy plate, and by using a section of glass tube with 
a sharp corner that sets the contact line location. This mitigates the effect of contact angle variation (Cram and Haynes 1971).

\section{3}

\section{Modern force-balance techniques}

To eliminate the inside meniscus of the du Noüy ring, modern force-balance techniques have emphasized dipping and raising solid axisymmetric objects. These methods suffer from a high wetted area to wetted perimeter ratio that significantly increases the buoyancy correction. Some also require multiple simultaneous measurements.

Padday et al. (1975) reports surface tension of air-liquid interfaces measured with a vertical circular cylindrical rod. The force on the rod was found to increase as the rod is pulled from the liquid until it reaches a stable maximum, just like the ring. Surface tension was deduced from calculated meniscus shapes and the measured maximum pull without contact angle measurements or empirical corrections since the contact line occurs at the edge formed at the intersection the cylinder's flat end and curved surface. Similar axisymmetric calculations for meniscii hanging from vertical surface piercing wires have been completed (White and Tallmadge 1965; Huh and Scriven 1969; Hildebrand and Tallmadge 1970; Hartland and Hartley 1976). Withdrawn circular cones have also been analyzed for the case of zero contact angle (Padday 1978). The technique described in Sect. 4 follows the approach of Padday et al. but does not require a large buoyancy correction.

Other efforts, including some very recent, use a sphere as the working solid (Yarnold 1946; Scheuludko and Nikolov 1975; Huh and Mason 1976; Fiebner and Sonntag 1979; Gunde et al. 1995; Zhang et al. 1996). Spherical geometry alleviates the alignment problems that could render the axisymmetric assumption invalid for rings, vertical rods, or cones. Like the other axisymmetric geometries, the pull on the sphere has a local stable maximum as it is continually raised. However, experimental difficulties are encountered when a small $\left(0-10^{\circ}\right)$ contact angle is involved; a deficiency intrinsic to the spherical geometry (Bayramli and Mason 1982).

Elaborate non-force-balance techniques have been developed for measuring the surface tension of air-liquid interfaces. These include static droplet shape methods (Pallas and Harrison 1990), methods based on droplet oscillations (Tian et al. 1995), and differential bubble pressure methods (Holcomb and Zollweg 1992). There are also a host of other surface tension measuring techniques, some of considerable antiquity. These include, but by no means are limited to, the drop weight method, the capillary rise method, and various dynamic techniques. For a variety of reasons associated with contact angle uncertainty and necessary withdrawal of liquid samples, these methods are ill-suited for general-purpose in-situ tensiometry in arbitrary sized vessels with and without surfactants.

\section{3}

\section{Wilhelmy plate experiments}

This section presents experimental results for the detachment and zero-buoyancy (with receding meniscus created by plate withdrawal) versions of the Wilhelmy plate method. These two methods produce the most repeatable results because both employ a receding contact angle (La Mer and Robbins 1958;
Gaines 1960; Kawanishi et al. 1970; Pallas and Pethica 1983). The basis for the measurements is Eq. (1) with $\theta$ assumed close to zero, and $\sigma$ assumed constant along the wetted perimeter of the slide. In practice, $W_{\text {app }}$ is removed by zeroing the electronic balance. Neglecting the variation in $\theta$ at the plate ends is the main defect of this approach.

As a first attempt to obtain an accurate value of $\theta$, we exploited the two-dimensional meniscus-shape solution for fluid attached to a $90^{\circ}$ corner (Princen 1969). This provides a simple relationship between $h$ and $\theta$ far from the ends,

$h=\left(\frac{2 \sigma(1-\sin \theta)}{\Delta \rho g}\right)^{1 / 2}$.

In theory, one could measure $W_{\text {total }}$ and $h$ and then solve Eqs. (1) and (3) simultaneously for $\sigma$ and $\theta$. Although this approach seems superior to the assumption $\theta=0$, it fails because the liquid meniscus rises somewhat above the bottom of the plate (i.e. $h^{\prime}$ is not zero), thus the quantity $h$ is no longer the same in Eqs. (1) and (3). In addition, the contact line is necessarily bowed at the plate ends causing a variation in $h^{\prime}$ along the perimeter. Our attempts to use the measured $h$ to correct for $\theta$ produced surface tension results that were unrealistically high. This problem with a two-dimensional model of the Wilhelmy plate has been previously noted (Furlong and Hartland 1979).

For the plate aspect ratios and roughness in the present experiments, the meniscus detaches from the plate before a stable maximum pull is reached because of end effects. Frames from a high-speed video of meniscus-plate detachment are shown on Fig. 3. The images display approximately $60 \%$ of
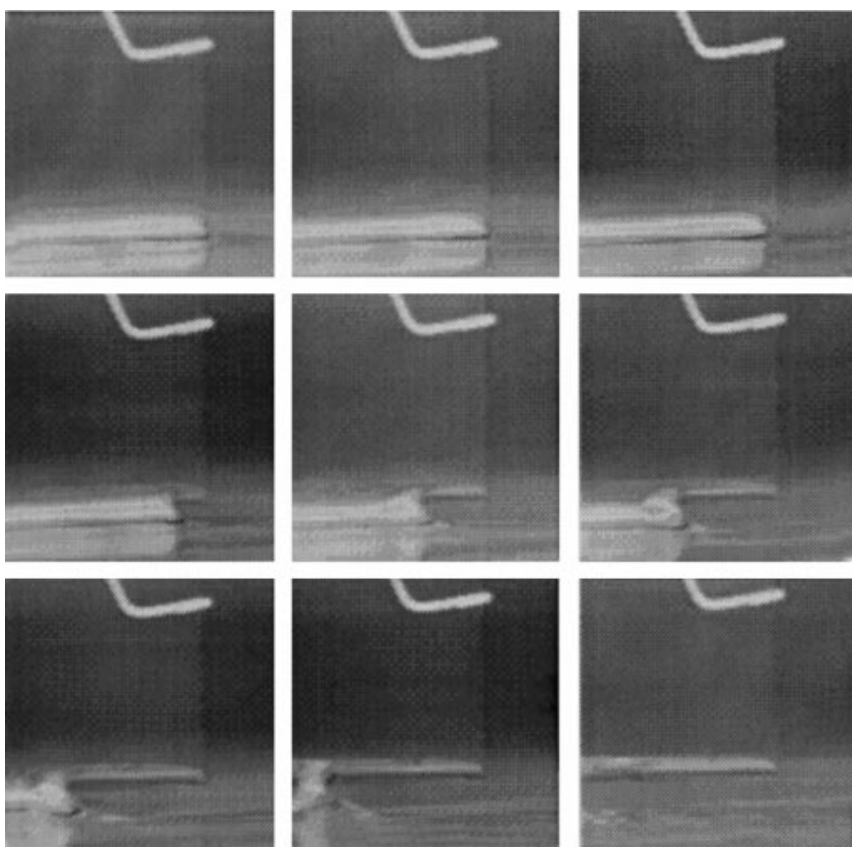

Fig. 3. Frames extracted from a high-speed video during detachment of a Wilhelmy plate. The sequence of frames goes left to right, top to bottom, with each frame separated by a time of $17.75 \mathrm{~ms}$. The sequence shows clearly that the meniscus detaches from the plate ends first 
the broadside view of the plate with approximately $18 \mathrm{~ms}$ between images. Meniscus rupture starts at the plate end and travels inward toward the center of the plate. If a twodimensional model of the plate's meniscus was valid, detachment would occur all at once at the entire plate perimeter. Figure 3 shows detachment to be governed by conditions at the plate ends. Therefore, meniscus properties must be different at the plate ends even before detachment (Pike and Bonnet 1970), so both detachment and zero-buoyancy methods must be influenced by conditions at the plate ends.

Two-dimensional modeling of the liquid meniscus fails because it neglects the second radius of curvature in the classic Young-Laplace equation (Defay and Prigogine 1966; Adamson 1990). Moreover, reducing plate thickness causes the second unaccounted for radius of curvature to become smaller, tending to decrease the pressure drop across the fluid interface. For hydrostatic equilibrium, this decrease in pressure can only be accommodated by a decrease in meniscus height $h^{\prime}$ which is consistent with the current and previous plate-end film-deficit observations. Therefore, as the plate is raised, the compound meniscus curvature at the plate ends forces the meniscus to slip off the plate end prematurely, and the entire meniscus ruptures short of two-dimensional prediction of maximum pull. Hence, decreasing plate thickness should promote premature rupture and lead to low surface tension measurements. This contention is supported in Loglio et al. (1976).

A investigation into the effect of plate thickness was conducted to quantify its importance in plate tensiometry. The emphasis here was to use thin plates to minimize the buoyancy correction. Measurements were taken with carbon- and particulate-filtered tap water (hereafter referred to as laboratory water), a commercial mixture of isopropyl alcohol $(70 \%)$ and water $(30 \%)$, and a solution of Triton X-100 ( (a soluble surfactant) and laboratory water in either a wave-tank $\left(7684 \mathrm{~cm}^{2}\right)$, or a circular Pyrex vessel $\left(410 \mathrm{~cm}^{2}\right)$ of sufficient size to render any finite container effect negligible (Furlong and Hartland 1979). The procedures employed were appropriate for a standard laboratory environment. Microscope cover slips [Corning No. 1 Cover Glass, $24 \times 50 \mathrm{~mm}$ (approximately $0.14 \mathrm{~mm}$ thick); Corning No. $1 \frac{1}{2}$ Cover Glass, $24 \times 50 \mathrm{~mm}$ (approximately $0.17 \mathrm{~mm}$ thick); Fisherbrand No. 2 Microscope Cover Glass, $24 \times 50 \mathrm{~mm}$ (approximately $0.21 \mathrm{~mm}$ thick)] were used as Wilhelmy plates and were only handled using new surgical gloves during mounting immediately prior to use. The working edge of the slip was never touched. No effort was made to change the roughness of the cover slip. The mounted plate was suspended by a hook beneath an electronic balance (AND model FX-400 with $410 \mathrm{~g}$ capacity and $1 \mathrm{mg}$ resolution). The cover slide was lowered to the liquid surface and its alignment was adjusted by observing its reflection (Pike and Bonnet 1970; Gaonkar and Neuman 1984). Once aligned, a receding contact angle was obtained by lowering the plate slightly beyond the point of contact with the interface and then bringing it back to its neutrally buoyant position. The measured pull was recorded at this point for the zero-buoyancy calculations. The plate was then quasi-statically raised in increments determined by $1 \mathrm{mg}$ increases on the electronic balance. The balance reading at meniscus detachment was recorded. The weight of the plate and adhering liquid after meniscus detachment was used as $W_{\text {app }}$ in the detachment technique calculations to get a more accurate measurement (Princen 1970; Lane and Jordan 1970; Lane and Jordan 1971). The surface temperature of the water and the vertical distance traversed by the plate above the free surface were recorded.

Experimental results for laboratory water, assuming $\cos \theta=1$, are shown on Fig. 4 where the vertical axis displays the measured surface tension divided by a tabulated surface tension value (accurate to $\pm 0.5 \%$ ) for pure water at the measured surface temperature (Gittens 1969), and the horizontal axis is the thickness to length ratio of the Wilhelmy plate. Although each measurement is repeatable, this figure shows that Wilhelmy plate measurements are plate-geometry dependent. This geometric dependence was also found with the other impure liquids. The observed trend follows the plate-end meniscus-rupture mechanics described above, and has been reported in previous investigations (Kawanishi et al. 1970; Gaonkar and Neuman 1984; Gaonkar and Neuman 1987). The geometry-induced variation in the values on Fig. 4 over the range of $t / L$ is approximately $\pm 1.5 \%$ for the detachment technique and nearly $\pm 4 \%$ for the zero-buoyancy technique. We conjecture that the lower results and greater variability using the zero-buoyancy technique result from the $\cos \theta=1$ assumption being more accurate for the detachment measurement.

\section{4}

\section{Annular slide method}

To circumvent the problem arising from the plate ends, axisymmetric slides fashioned from short sections of quartz tubing were used in place of the traditional rectangular Wilhelmy plates (see Fig. 5). Slowinski and Masterton (1961)

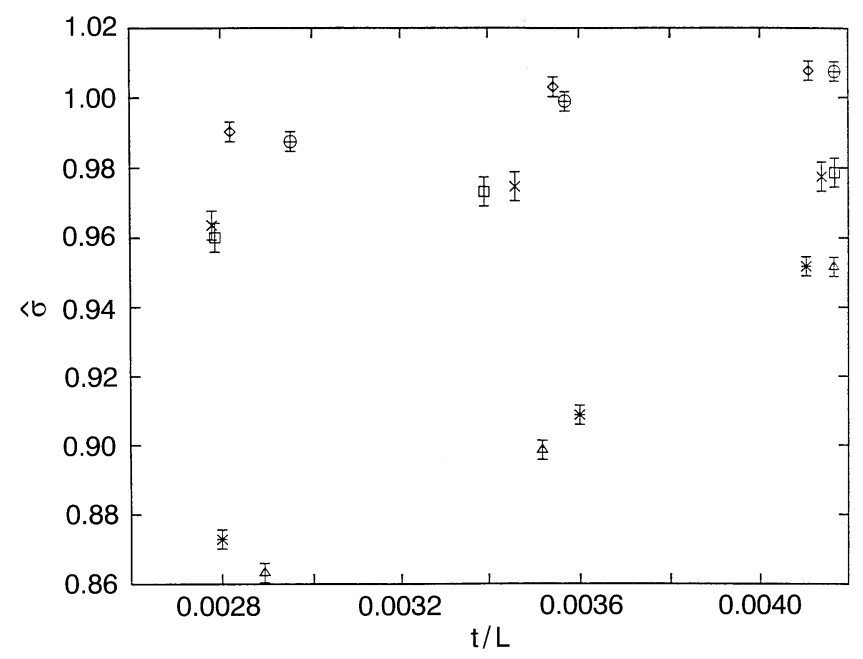

Fig. 4. Surface tension measurements versus thickness for same length Wilhelmy plates. Measured surface tension scaled by a reference surface tension at the measured surface temperature from Gittens (1969) is represented by $\hat{\sigma}$. Some of this data was acquired using the detachment method and reduced with both the simple model and the buoyancy-corrected model. The simple model $(\diamond, \oplus)$ uses (2) and the buoyancy-corrected model $(\square, \times)$ uses (1). The zerobuoyancy method with a receding-meniscus technique $(*, \Delta)$ is also presented using (2). Different symbols for the same method indicate data taken on different days. All cases assume $\cos \theta=1$ along the entire perimeter. The error bars indicate calculated uncertainties 

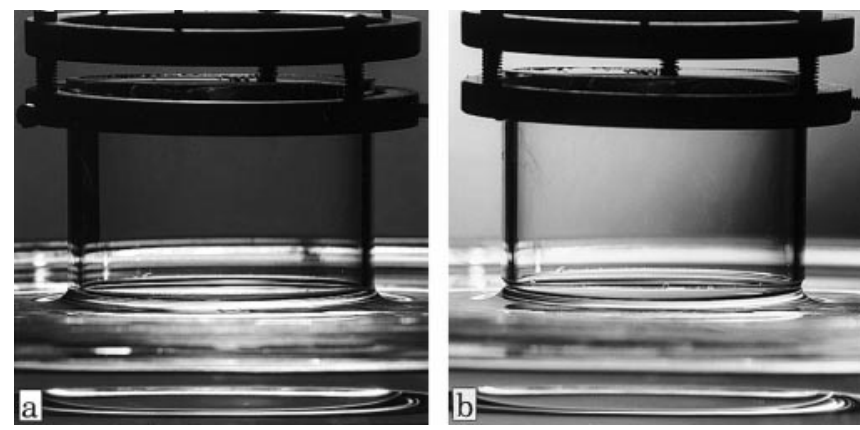

Fig. 5. The annular slide before detachment. The height of the slide above the undisturbed free surface is greater in $\mathbf{b}$ than in $\mathbf{a}$. The under-cutting of the outer meniscus is captured well on the righthand-side of $\mathbf{b}$. While a photograph depicting the under-cutting of the inner meniscus is impossible to obtain, both meniscii are predicted to be under-cutting at the time of measurement (when the maximum pull is reached) for the geometry shown. The slide is never detached from the surface during measurement

presents a brief account of surface tension measurements using a thin platinum sheet wrapped into a tubular geometry.

However, meniscus shape computations were not pursued and curvature effects were ignored. Commercial versions of this rudimentary circular Wilhelmy plate are available. Independently, a recent monograph (Rusanov and Prikhorov 1996) refers to a technique involving a hollow cylinder but meniscus calculations are not pursued. An approximation is presented for the case where both the inside and outside angles with the vertical are assumed equal to zero. We found that using the simple-model approximations commonly adopted by Wilhelmy plate users of contact angles equal to zero and neglecting the buoyancy correction cause $\pm 20 \%$ errors for typical annular slide geometries. In this section, numerical meniscus shape results are reduced to a convenient form via the use of dimensionless variables.

Unlike microscope cover slips, our annular slides were fashioned by hand. After being cut from quartz tubes of various sizes and wall thicknesses, one end of the slide was ground flat and smooth to the touch. Care was taken to insure right angles between the cut surface and smooth sides of the tube. Quartz was chosen for the annular slide material because standard quartz tube stock is inexpensive, readily available, inert, and easy to clean. The mechanical stability of the quartz tube stock prohibited use of thicknesses as small as those obtained with glass Wilhelmy plates. However, the relatively small increase in the buoyancy correction in percentage terms does not diminish the accuracy. Beyond force and geometry measurements, an axisymmetric meniscus theory is all that is required for the annular slide method.

A cross-sectional schematic for the axisymmetric model of the annular slide is shown in Fig. 6 and the annular slide counterpart of Eq. (1) is

$W=W_{\text {total }}-W_{\text {app }}=\left(P_{0} \cos \theta_{0}+P_{i} \cos \theta_{i}\right) \sigma+\Delta \rho g \pi\left(R_{0}^{2}-R_{i}^{2}\right) h$

where $\theta_{0}, h, \theta_{i}$, and $\sigma$ are determined numerically. For these hydrostatic calculations, it is assumed that the maximum pull occurs before meniscus rupture and that the inner and outer

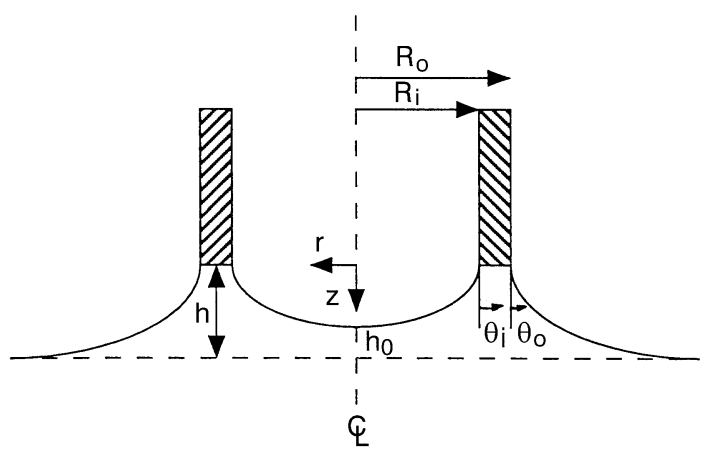

Fig. 6. Cross-sectional schematic of the annular slide geometry using an axisymmetric coordinate system. For the annular slides used in this study, $R_{i}$ was sufficiently large such that $h_{0} \approx 0$

meniscii can be computed separately. The maximum pull was predicted and observed to occur with both meniscii undercutting (where $\theta_{i}$ is positive and $\theta_{0}$ is negative in Fig. 6 , the right side meniscus in Fig. 5b depicts under-cutting of the outer meniscus). Therefore, it is necessary to monitor whether the inner and outer meniscii would come into contact to avoid physically unrealizable solutions or so close as to cause rupture by molecular forces.

The hydrostatic pressure equation can be written as

$p=\Delta \rho g(z-h)$.

Using parametric representations for the meniscus coordinates [ $r(s)$ and $z(s)$, where $s$ is the arclength along the meniscus], the Young-Laplace equation for the pressure difference across the interface as a result of surface tension becomes:

$p= \pm \sigma\left[\frac{\left(1-r^{\prime 2}\right)^{1 / 2}}{r}-\frac{r^{\prime \prime}}{\left(1-r^{\prime 2}\right)^{1 / 2}}\right]$

where the prime denotes differentiation with respect to $s$. The positive right-hand side is for the outside meniscus, and the negative is for the inside meniscus.

Combining Eqs. (5) and (6) forms a second-order ODE for $r$ that is solved simultaneously with

$z^{\prime}=\left(1-r^{\prime 2}\right)^{1 / 2}$.

The boundary conditions on the outside meniscus are

$r=R_{0}, \quad z=0 \quad$ at $s=0$

and

$r^{\prime}=1, \quad z=h \quad$ as $s \rightarrow \infty$.

The last additional boundary condition results from the unknown $h$ in Eq. (4). On the inside meniscus the boundary conditions are

$r=R_{i}, \quad z=0 \quad$ at $s=0$

and

$r^{\prime}=1 \quad$ at $r=0$,

with no extra boundary condition for $z$ on the inside because $h$ is determined from the outside meniscus. 
The system of equations can be solved using standard shooting techniques, starting with the outside meniscus. Shooting parameters $h$ and $r^{\prime}(0)=\sin \theta_{0}$ are systematically chosen to satisfy the two boundary conditions at infinity. Upon completion, the inside meniscus is solved with one shooting parameter $r^{\prime}(0)=\sin \theta_{i}$. For the $R_{i}$ considered here, $r^{\prime \prime}(0) \gg R_{i}^{-1}$ and the inside meniscus becomes flat near $r=0$. This leads to $z \rightarrow h$ or $h_{0}=0$ for the solutions presented here.

To experimentally implement the annular slide method one determines the inner and outer tube radii and measures the maximum pull on the slide that implicitly determines values of $\theta_{0}, h, \theta_{i}$, and $\sigma$. Figure 7 shows a typical relationship between $h$ and the pull on the slide. Measurements for this slide geometry are also included on this figure. Note that the smallest three abscissa values begin to drift from the theoretical curve. Here the outer meniscus is attached to the corner and under-cutting, however, the inner meniscus may not have reached the corner allowing a nonzero interior $h^{\prime}$. The calculation is based on both menisci attached to the corners. However, the match is excellent near $W_{\max }$, where the measurements are made, and past $W_{\max }$ to the point of meniscus rupture. The material properties of the slide do not influence the results, so any inert solid material wetted by the test liquid should be acceptable.

The results of the meniscus calculations can be cast into a more useful algebraic form using dimensionless variables. Although it has become customary to use Laplace's capillary length for scaling meniscus problems, it is advantageous here to have the surface tension $\sigma$ appear in only one parameter as this is the unknown quantity. Therefore, the following three dimensionless parameters were used: (i) an aspect ratio that

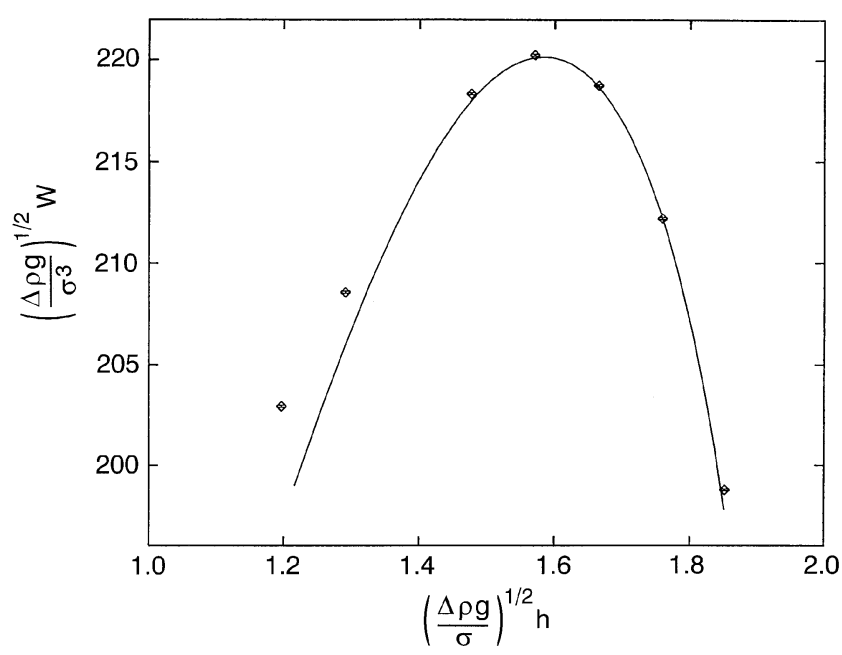

Fig. 7. Computations and measurements of $W$ as a function of $h$ (as well as $\theta_{0}$ and $\theta_{i}$ ) for a annular slide with $2 R_{0}=59.9 \mathrm{~mm}$ and $R_{i}=55.2 \mathrm{~mm}$. The axes are non-dimensionalized with the density difference between the liquid and air, gravity, and surface tension just as Laplace originally non-dimensionalized meniscus problems (see Princen 1969). The annular-slide measured surface tension is used to scale the computed curve. The peak of this curve, termed the maximum pull, is the lone measurement required during experimental procedures to implement the annular slide method. Disagreement at low abscissa values is the likely result of the interior $h^{\prime}$ (see Fig. 1) being nonzero, a value unaccounted for in the computational curve depends only on the annular geometry, $\tilde{t}=\left(R_{0}-R_{i}\right) / \bar{R}$ where $\bar{R} \equiv\left(R_{0}+R_{i}\right) / 2$ is the length scale, ii) a scaled maximum pull $\tilde{W}=W_{\max } /\left(\Delta \rho g \bar{R}^{3}\right)$, and iii) an inverse Bond number $\tilde{\sigma}=\sigma /\left(\Delta \rho g \bar{R}^{2}\right)$. Figure 8 shows the relationship $\bar{W}$ versus $\tilde{\sigma}$ for constant $\tilde{t}$. For the anticipated range of practical values, $0.05 \leqslant \tilde{t} \leqslant 0.1$ (including the specific $\tilde{t}$ for our three annular slides: tube A; $2 R_{0}=59.89 \mathrm{~mm}, 2 R_{i}=55.16 \mathrm{~mm}$, GM Associates, Inc. of Oakland, CA stock \# 6000-67: tube B; $2 R_{0}=$ $53.85 \mathrm{~mm}, 2 R_{i}=49.46 \mathrm{~mm}$, GMA stock \# 6000-63: tube C; $2 R_{0}=49.87 \mathrm{~mm}, 2 R_{i}=46.72 \mathrm{~mm}$, GMA stock \# 6000-61), and the $\tilde{\sigma}$ range shown, this relationship is well approximated (relative error less than $\pm 0.2 \%$ ) by the bi-cubic polynomial:

$$
\begin{aligned}
\tilde{\sigma}= & 0.00033967-0.026690 \tilde{t}+0.071559 \tilde{W}+0.18044 \tilde{t}^{2} \\
& +0.058571 \tilde{W}^{2}-0.40662 \tilde{t} \tilde{W}-0.21415 \tilde{t}^{3}-0.11936 \tilde{W}^{3} \\
& +0.15006 \tilde{t}^{2} \tilde{W}+0.41917 \tilde{t} \tilde{W}^{2}
\end{aligned}
$$

Hence, measurements of $\tilde{t}$ and $\tilde{W}$ can be used to determine $\tilde{\sigma}$, and thus the surface tension. Measurements of the inner and outer diameter to \pm 10 microns, and weight to $\pm 1 \mathrm{mg}$ results in a calculated error of \pm 0.12 dyne/cm when using Eq. (12) for a liquid of unit specific gravity and surface tension near that of pure water. The error drops as a function of measured surface tension such that the percent error in measurement remains the same.

The procedures followed in making surface tension measurements with the annular slide vary little from those

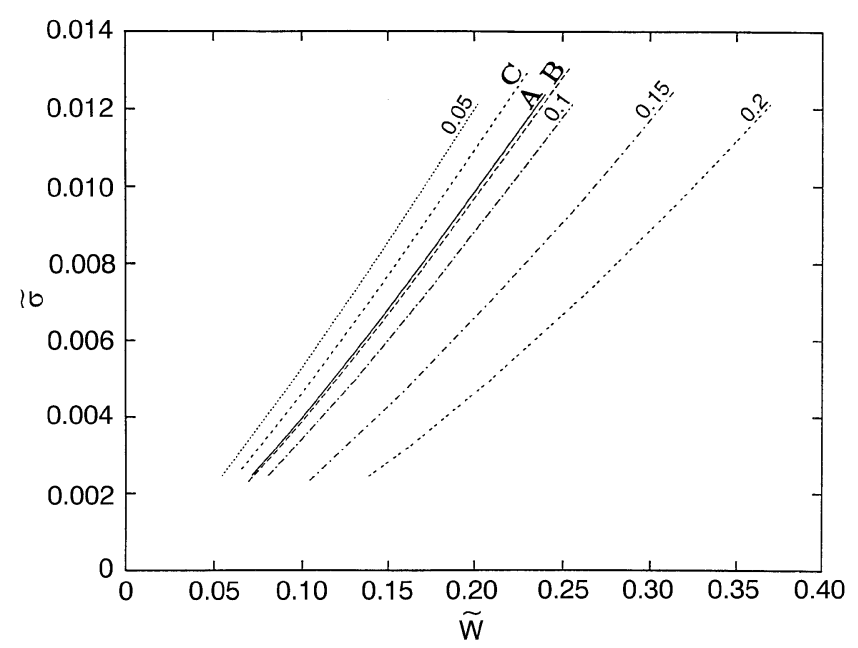

Fig. 8. Computations of $\tilde{\sigma}$ as a function of $\tilde{W}$ for various values of $\tilde{t}$. The range of $\tilde{t}$ includes the three annular slides used in the experiments (tube A, $\tilde{t}=0.08218$; tube $\mathrm{B}, \tilde{t}=0.08487$; tube $\mathrm{C}$, $\tilde{t}=0.06520)$, but does not cover the entire possible span $(0 \leqslant \tilde{t} \leqslant 2)$. The $\tilde{t} \rightarrow 0$ case will not experience a maximum pull. The $\tilde{t}=2$ case represents a solid circular rod as in Padday et al. (1975). Our calculations recover the results of Padday et al. (1975), although this data is not shown. The uncalculated regions near the origin represent cases where the absolute surface tension is less than 15.0 dyne $/ \mathrm{cm}$. We conjecture $\tilde{\sigma}$ goes to zero as $\tilde{W}$ goes to zero, however there is a possibility that solutions do not exist near the origin. The monotonic trends in the curves with respect to $\tilde{t}$ continue to the limit $\tilde{t}=2$. For the displayed data in the range of $\tilde{t}=0.05$ to $\tilde{t}=0.1$, the polynomial of Eq. (12) approximates $\tilde{\sigma}$ to within $\pm 0.2 \%$ 
previously described for Wilhelmy plates with two exceptions: 1) meniscus detachment and/or monitoring of $h$ was not necessary, and 2) the bottom plane of the annular slide was aligned with the liquid surface using a special harness with thumb screw adjustments. This alignment was obtained by ensuring uniform contact between the slide and a leveled mirror surface. An additional indication of slide alignment is provided by the uniformity of the dynamic wetting of the slide when it first touches the test liquid. Ordinary hygienic preparation of the slides was sufficient to obtain repeatable results under the existing laboratory conditions. The slide was washed thoroughly in detergent and hot tap water, rinsed in hot tap water for ten minutes, and then rinsed for ten minutes and boiled in laboratory water. Just before measurement, the slide was removed from the last continuous rinse and placed working-edge up on a hot plate insuring quick evaporation of water and minimal residue.

Once the dry annular slide was aligned in its harness, the balance was zeroed to remove $W_{\text {app }}$. The annular slide was then lowered into contact with the liquid. As the annular slide is quasi-statically raised, the force continuously increases until $W_{\max }$ is reached as shown on Fig. 7. This maximum is stable and not subject to hysteresis as the slide is raised or lowered. At the point of maximum pull, the outer meniscus was observed to hang from the corner of the slide and undercut the bottom face of the slide, in agreement with the axisymmetric analysis and the previous efforts with wetted cylinders (Padday et al. 1975). Here, the undercut ensures that $h^{\prime}$ (see Fig. 1) is zero. Unlike the detachment version of the Wilhelmy plate, there is no need to account for any liquid remaining on the annular slide since its sides are not wet at the point of maximum pull as was previously found for solid, cylindrical rod tensiometry (Padday et al. 1975).

Figure 9 shows scaled laboratory-water surface-tension measurements from our three annular slides. Figure 10 shows measured results for two other impure liquids having surface tensions significantly different from that of water. These plots show the geometric independence of the annular slide measurements as compared to the Wilhelmy plate results on Fig. 4. In addition, the results on Fig. 9 are within $1 \%$ of the tabulated results for pure water at the same temperature, meeting expectations for our laboratory conditions and water purity. The data scatter is the best estimate of the technique's uncertainty as implemented here. The overall variability of results across geometries has been reduced to $\pm 0.5 \%$, one third that of the best version of the Wilhelmy plate. Further reduction in annular slide measurement uncertainty is certainly possible with a higher precision balance, better slide manufacturing, precision metrology, and improved laboratory hygiene.

Figure 11 shows the influence of small tilt angles $(\alpha)$ on annular slide results. The more or less quadratic dependence on tilt angle matches previous experimental results for the du Noüy ring (Harkins and Jordan 1930), and suggests that small tilt angles, less than half a degree are not significant. Hence, acceptable results can be obtained via leveling with an ordinary carpenter's level.

For completeness, zero-buoyancy with receding meniscus measurements were performed on the laboratory water with the annular slide assuming $\cos \theta_{0}=\cos \theta_{i}=1$ while monitoring

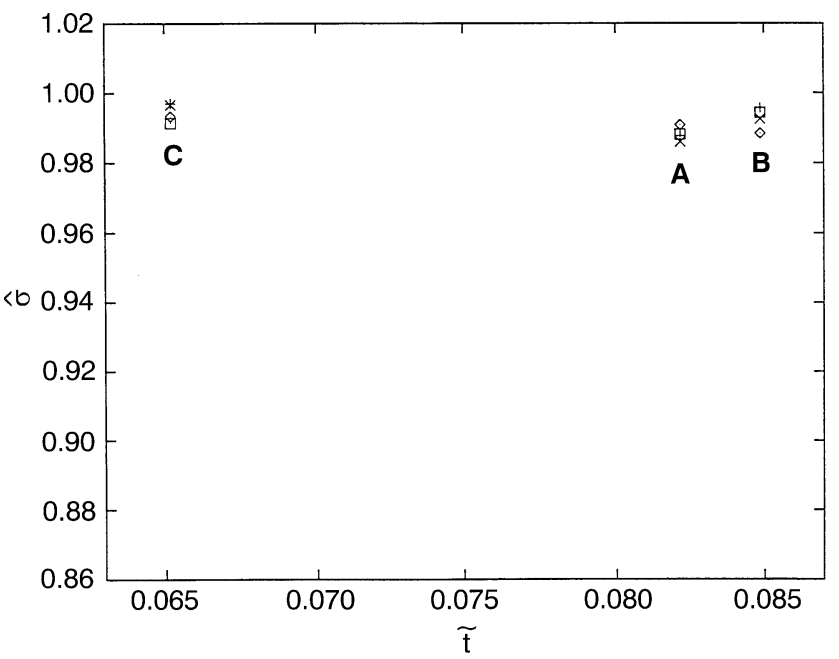

Fig. 9. Measurements of surface tension with three annular slides $(A$, $B, C)$ scaled by a reference surface tension at the measured surface temperature versus dimensionless slide thickness $\tilde{t}$. Like symbols indicate data taken on the same day. The data are plotted on the same vertical scale as Fig. 4 for comparison. Statistical scatter shows the annular slides produce more consistent measurements than Wilhelmy plates of varying thickness. The calculated error based on uncertainty of quantities involved in the measurement is approximately $\pm 0.4 \%$, however, the error bars were omitted for clarity

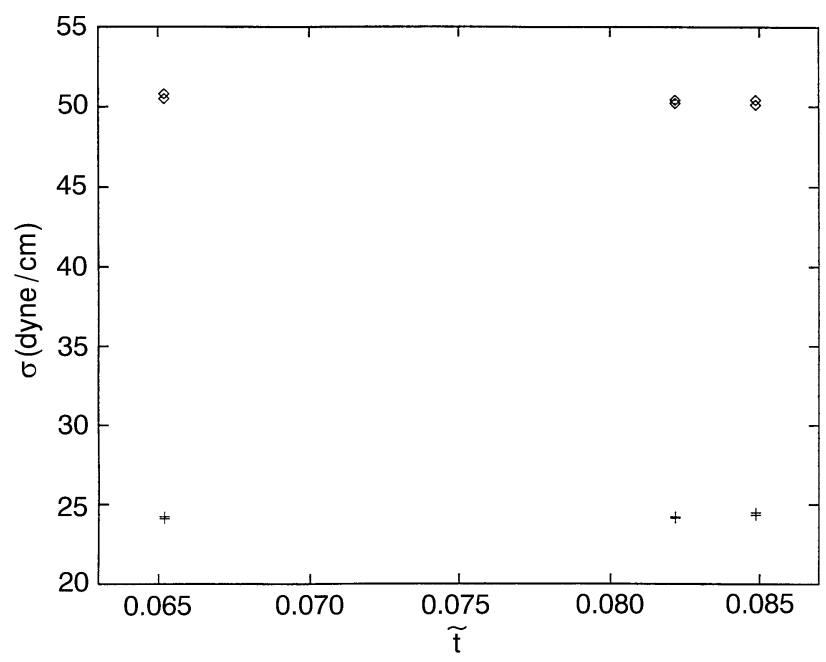

Fig. 10. Annular slide measurements of surface tension for two impure liquids versus $\tilde{t}$. The values near $50 \mathrm{dyne} / \mathrm{cm}$ were obtained from a solution of laboratory water and Triton X-100 ( $)$ (a soluble surfactant). The values near 25 dyne/cm were obtained from a commercial mixture of isopropyl alcohol $(70 \%)$ and water $(30 \%)$. Along with Fig. 9, these measurements show the annular slide technique to produce results that are independent of $\tilde{t}$

the $h=0$ level (in effect, having to measure an additional quantity). The results showed almost eight times the scatter but with the same mean as the maximum-pull results displayed on Fig. 9.

In summary, the annular slide conforms better to an axisymmetric theory than the Wilhelmy plate does to a 


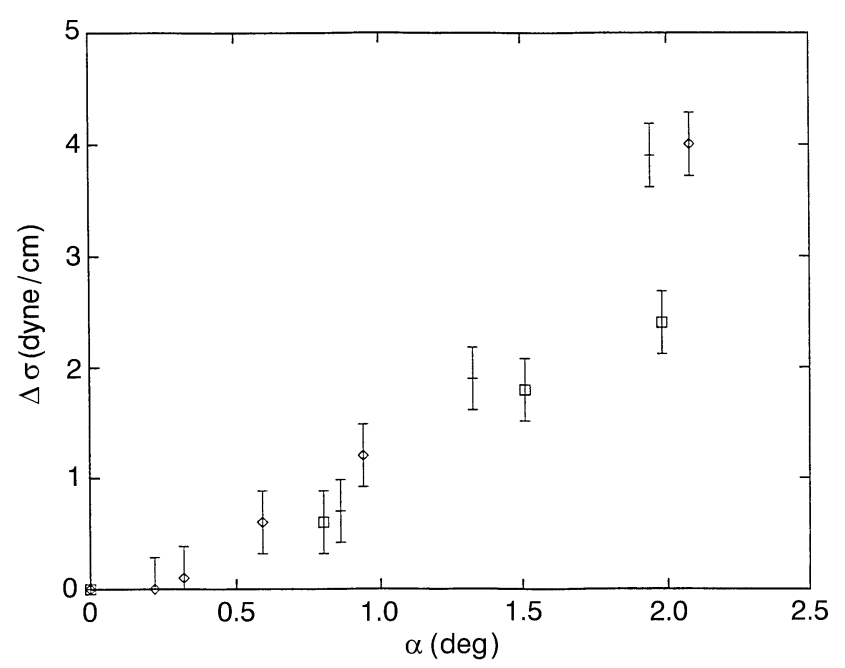

Fig. 11. Laboratory water surface tension measurement variation with tilt angle $(\alpha)$. The vertical axis is the difference between the surface tension measured with zero tilt minus the surface tension measured with a known tilt. The annular slide method is relatively insensitive to tilt angles less than half a degree and the measurement error is essentially proportional to the tilt angle squared. Different symbols correspond to annular slides with different values of $\tilde{t}(A=\diamond, B=+$, $C=\square)$

two-dimensional theory. This renders the annular slide results independent of slide thickness.

\section{5}

\section{Summary and conclusions}

The extensive literature on surface tensiometry still contains many contradictions and contentious issues. While their accuracy varies, in-situ force-balance tensiometry methods can be classified according to the number of measurements and assumptions required. All methods assume the gravitational acceleration, the air-liquid density difference, and the defining geometric lengths are known. The simplest version of the Wilhelmy plate, vertical rods, and the annular slide require only one force measurement. The Wilhelmy plate with buoyancy correction, du Noüy ring, and spheres require at least two measurements, or one measurement and one assumption. Usually the additional measurement or assumption concerns the contact angle or the height at maximum pull, both notorious for causing inaccuracies.

The known meniscus contact location of the rod and the annular slide sets these techniques apart and removes the error from assuming the contact line moves smoothly along an object (like a ring or sphere) with the contact angle remaining constant. Although moving contact lines (Dussan and Davis 1974; Dussan 1976, 1979) are beyond the scope of this paper, a stationary contact line is preferable for general purpose tensiometry.

The methods requiring only one measurement also differ. The Wilhelmy plate method without buoyancy correction and the assumption $\cos \theta=1$ produces results that depend on plate thickness. The vertical rod geometry is simpler than the annular slide geometry in that the rod has no interior meniscus. However, the contribution to the pull from the buoyancy force compared to the contribution from the surface tension force is much higher for rods than for tubes. Padday et al. (1975) showed large scatter as the rod radius was increased and that very sensitive measurements of pull and rod radii were required for smaller rods. While proper alignment of rings, plates, rods and annular slides is a nontrivial part of these methods, tilt corrections are small and slide alignment is manageable with modest means.

Our dissatisfaction with the existing tensiometry methods has fostered the development of a self-consistent in situ force-balance technique free of empirical corrections and calibrations. The annular-slide technique produces reliable results across geometric variations, and yields a root-meansquare uncertainty approaching $\pm 0.1 \mathrm{dyne} / \mathrm{cm}$ with only a modest amount of equipment (annular slide, harness, milligram balance, and uncalibrated vertical traverse). While the annular slide is similar to other force-balance techniques, it gleans their best attributes for robust tensiometry with unknown and impure liquids in an ordinary laboratory environment. It can also be readily extended to precision measurements of pure liquids, and continuous surface tension measurements with appropriate refinements.

\section{References}

Adamson A (1990) Physical chemistry of surfaces. New York: Wiley Allan A (1958) Wilhelmy's plate and Young's equation. J Colloid Sci 13: 273

Bayramli E; Mason S (1982) Some comments on sphere tensiometry. Colloid Polymer Sci 260: 452

Cini R; Loglio G; Ficalbi A (1972) Temperature dependence of the surface tension of water by the equilibrium ring method. J Colloid Interface Sci 41: 287

Cram P; Haynes J (1971) The influence of contact angle on surface tension measurements by the ring detachment method. J Colloid Interface Sci 35: 706

Davies J; Rideal E (1963) Interfacial phenomena. New York: Academic Press

Dussan E; Davis S (1974) On the motion of a fluid-fluid interface along a solid surface. J Fluid Mech 65: 71

Dussan E (1976) The moving contact line: the slip boundary condition. J Fluid Mech 77: 665

Dussan E (1979) On the spreading of liquids on solid surfaces: static and dynamic contact lines. Ann Rev Fluid Mech 11: 371

Fieber C; Sonntag H (1979) Theoretical consideration on the applicability of the sphere method for measuring interfacial tension and contact angle. Colloid Polymer Sci 257: 874

Freud B; Freud H (1930) A theory of the ring method for the determination of surface tension. J Amer Chem Soc 52: 1772

Furlong D; Hartland S (1979) Wall effect in the determination of surface tension using a Wilhelmy plate. J Colloid Interface Sci 71: 301

Furlong D; Freeman P; Metcalfe I; White L (1983) Wall effects in du Noüy ring tensiometry. J Chem Soc Faraday Trans I 79: 1701

Gaines G (1960) Some observations on monolayers of carbon-14 labeled stearic acid. J Colloid Sci 15: 321

Gaines G (1966) Insoluble monolayers at liquid-gas interfaces. New York: Interscience Publishers

Gaonkar A; Neuman R (1984) The effect of wettability of Wilhelmy plate and du Noüy ring on interfacial tension measurements in solvent extraction systems. J Colloid Interface Sci 98: 112

Gaonkar A; Neuman R (1987) The uncertainty in absolute values of surface tension of water. Colloids Surfaces 27: 1

Gaonkar A; Neuman R (1991) Comment on "The surface tension of water". Colloids Surfaces 61: 353

Gifford W (1978) The effect of contact angle on ring tensiometry. J Colloid Interface Sci 64: 588 
Gittens G (1969) Variation of surface tension of water with temperature. J Colloid Interface Sci 30: 406

Gunde R; Hartland S; Mäder R (1995) Sphere tensiometry: a new approach to simultaneously and independent determination of surface tension and contact angle. J Colloid Interface Sci 176: 17

Harkins W; Jordan H (1930) A method for the determination of surface and interfacial tension from the maximum pull on a ring. J Amer Chem Soc 52: 1751

Harkins W; Anderson T (1937) A simple accurate film balance of the vertical type for biological and chemical work, and a theoretical and experimental comparison with the horizontal type. J Amer Chem Soc 59: 2189

Hartland S; Hartley R (1976) Axisymmmetric fluid-liquid interfaces. New York: Elsevier Scientific Publishing

Hildebrand M; Tallmadge J (1970) Corrected values for static menisci on the outside of cylinders. J fluid mech 44: 811

Holcomb C; Zollweg J (1992) Improved differential bubble pressure surface tensiometer. J Colloid Inteface Sci 154: 51

Huh C; Scriven L (1969) Shapes of axisymmetric fluid interfaces of unbounded extent. J Colloid Interface Sci 30: 323

Huh C; Mason S (1975) A rigorous theory of ring tensiometry. Colloid and Polymer Sci 253: 566

Huh C; Mason S (1976) Sphere tensiometry: an evaluation and critique. Can J Chem 54: 969

Huh C; Mason S (1977) A rigorous theory of ring tensiometry: addendum on the wall effect. Colloid Polymer Sci 255: 460

Ip S; Toguri J (1994) The equivalency of surface tension, surface energy and surface free energy. J Mater Sci 29: 688

Jordan D; Lane J (1964) A thermodynamic discussion on the use of a vertical-plate balance for the measurement of surface tension. Aust J Chem 17: 7

Kawanishi T; Seimiya T; Sasaki T (1969) Some remarks on surface tension measurements by the Wilhelmy method using a tilted or roughened plate. Aust J Chem 22: 2247

Kawanishi T; Seimiya T; Sasaki T (1970) Corrections for surface tension measured by Wilhelmy method. J Colloid Interface Sci 32: 622

La Mer V; Robbins M (1958) The effect of the spreading solvent on the properties of monolayers. J Phys Chem 62: 1291

Lane J; Jordan D (1970) The measurement of surface tension by means of a vertical-plate balance. Aust J Chem 23: 2153

Lane J; Jordan D (1971) Further comment on the vertical-plate balance. Aust J Chem 24: 1297

Loglio G; Ficalbi A; Cini R (1976) On the theory of equilibrium maximum pull methods for the measurement of the surface tension of liquids. J Colloid Interface Sci 56: 383

Mennella A; Morrow N (1995) Point-by-point method of determining contact angles from dynamic Wilhelmy plate data for oil/brine/solid systems. J Colloid Interface Sci 172: 48

Orr F; Scriven L; Rivas A (1975) Menisci in arrays of cylinders: Numerical simulation by finite elements. J Colloid Interface Sci 52: 602

Orr F; Scriven L; Chu T (1977) Menisci around plates and pins dipped in liquid: Interpretation of Wilhelmy plate and solderability measurements. J Colloid Interface Sci 60: 402

Padday J (1957) A direct reading electrically operated balance for static and dynamic surface tension measurement. In: Proc 2nd Intern Congr Surface Activity. Vol 1, London: Butterworth, pp 1-6

Padday J; Russell D (1960) The measurement of the surface tension of pure liquids and solutions. J Colloid Sci 15: 503

Padday J (1969) Surface tension. In: Surface and colloid science. (ed Matijevíc E). Vol 1, New York: Wiley-Interscience, pp 39-149

Padday J; Pitt A; Pashley R (1975) Menisci at a free liquid surface: surface tension from the maximum pull on a rod. J Chem Soc Faraday Trans I 71: 1919

Padday J (1978) Menisci formed by a cone at a free liquid surface: An absolute method of surface tension measurement. J Chem Soc Faraday Trans I 74: 2827
Pallas N; Pethica B (1983) The surface tension of water. Colloids Surfaces 6: 221

Pallas N; Pethica B (1989) The surface tension of water. Colloids Surfaces 36: 369

Pallas N; Harrison Y (1990) An automated drop shape apparatus and the surface tension of pure water. Colloids Surfaces 43: 169

Pallas N; Pethica B (1991) A reply to A.G. Gaonkar and R.D. Neuman on "The surface tension of water". Colloids Surfaces 61: 355

Pike F; Bonnet J (1970) The end-correction in the Wilhelmy technique for surface tension measurements. J Colloid Interface Sci 34: 597

Princen H; Mason S (1965) Shape of a fluid drop at a fluid-liquid interface: II. Theory for three-phase systems. J Colloid Sci 20: 246

Princen H (1969) The equilibrium shape of interfaces, drops, and bubbles. Rigid and deformable particles at interfaces. In: Surface and colloid science (ed Matijevíc E). Vol. 2, New York: WileyInterscience, pp 1-84

Rusanov A; Prokhorov V (1996) Interfacial tensiometry. Amsterdam: Elsevier Science

Sauer B; Carney T (1990) Dynamic contact angle measurements on glass fibers: Influence of fiber diameter on hysteresis and contact line pinning. Langmuir 6: 1002

Scheludko A; Nikolov A (1975) Measurement of surface tension by pulling a sphere from a liquid. Colloid Polymer Sci 253: 396

Slowinski E; Masterton W (1961) A simple absolute method for the measurement of surface tension. J Phys Chem 65: 1067

Taylor J; Mingins J (1975) Properties of the non-polar oil/water interface, Part I. J Chem Soc Faraday Trans I 71: 1161

Tian Y; Holt R; Apfel R (1995) Investigations of liquid surface rheology of surfactant solutions by droplet shape oscillations: Theory Phys Fluids 7: 2938

White D; Tallmadge J (1965) Static menisci on the outside of cylinders. J Fluid Mech 23: 325

Yarnold G (1946) The hysteresis of the angle of contact of mercury. Proc Phys Soc 58: 120

Zhang L; Ren L; Hartland S (1996) More convenient and suitable methods for sphere tensiometry. J Colloid Interface Sci 180: 493

Zotova K; Trapeznikov A (1960) The plate equilibrium method for studying the surface tension of solutions of semi-colloidal substances during the slow attainment of equilibrium. Russ J Phys Chem 34: 93 